bucil $^{6}$ and chronic active hepatitis treated with azathioprine. ${ }^{7}$ Cryptococcal meningitis has also been reported in apparently otherwise healthy people. ${ }^{8}$

The clinical manifestations include those of acute meningitis, or more commonly of chronic slow-onset meningitis. Severe psychiatric disturbances may occur. ${ }^{9}$ The cerebrospinal fluid pressure is usually increased, as is also the protein concentration of the spinal fluid. The cellular content may vary from two or three cells to over $1000 / \mathrm{mm}^{3}$, with a predominantly lymphocytic response. The classical method for detecting the cryptococci is to add a drop of Indian ink to a drop of the spinal fluid and then examine the mixture under the microscope, when the yeasts are seen with their surrounding capsules. Every attempt should be made to culture the organism so that it may be tested for its susceptibility to antifungal agents. Unfortunately the results of direct microscopy or culture are not always reliable, and the introduction of the latex agglutination test for detecting cryptococcal antigen $^{1011}$ has improved the diagnostic accuracy. A titre of $1 / 8$ or more should be regarded as an indication for treatment. If repeated tests on samples of lumbar spinal fluid give negative results and there is a strong clinical suspicion of cryptococcal meningitis the cisternal fluid should also be examined. ${ }^{12} \mathrm{Gas}$ chromatography has been used in the diagnosis of cryptococcal meningitis, ${ }^{13}$ but its role requires further evaluation.

Cryptococcal infections may also occur in other parts of the body: the lungs, ${ }^{14-16}$ larynx, ${ }^{17}$ eyes, ${ }^{18}$ lymph nodes, ${ }^{19}$ bone marrow, ${ }^{20}$ and skin. ${ }^{72}$ The diagnosis of these infections mainly depends on the results of histological examination and of culture of biopsy specimens. Stains used should include Grocott's methenamine silver, periodic acid-Schiff, and Mayer's mucicarmine. The organism may also be differentiated in histological sections by immunofluorescence. ${ }^{6}$

Treatment of cryptococcal infections became possible with the introduction of amphotericin B. ${ }^{22}$ Unfortunately the side effects were, and still are, a deterrent to both patients and doctors. The introduction of 5-fluorocytosine (5FC) ${ }^{2324}$ was regarded as a breakthrough in treatment, but it soon became apparent not only that naturally resistant strains occurred but also that resistance could emerge during treatment.

Fortunately, amphotericin B and 5FC may be used together ${ }^{2526}$ and reduce the emergence of 5FC-resistant strains, enabling lower doses of amphotericin $\mathrm{B}$ to be given. If the infecting strain is fully resistant to $5 \mathrm{FC}$ amphotericin $\mathrm{B}$ should be used alone in the usual dose. Hence all isolates of crytococci should be tested for their susceptibility to 5FC. Nevertheless, successful treatment of cryptococcal disease depends not only on the use of antifungal drugs but also on early recognition and treatment of the infection.

${ }^{1}$ Emmons, C W, Public Health Reports, 1960, 75, 362.

2 Gendel, B R, Ende, M, and Norman, S L, American fournal of Medicine, 1950, 9, 343.

${ }^{3}$ Khan, M A, and Sbar, S, Postgraduate Medical fournal, 1975, 51, 660.

4 Speller, D C E, et al, fournal of Clinical Pathology, 1977, 30, 254.

5 Whitley, T H, Graybill, J R, and Alford, R H, Southern Medical fournal, 1976, 69, 33.

${ }^{6}$ Wolf, P L, American fournal of Pathology, 1975, 78, 17a

7 Gauder, J P, Fournal of the American Medical Association, 1977, 237, 672.

8 Watkins, J S, et al, British Medical fournal, 1969, 3, 29.

${ }^{9}$ Martin, R A, Bates, D, and Shaw, D A, British Medical fournal, 1975, 3, 75.

${ }^{10}$ Bloomfield, N, Gordon, M A, and Elmendorf, D F, jun, Proceedings of the Society for Experimental Biology and Medicine, 1963, 114, 64.

11 Snow, R M, and Dismukes, W E, Archives of Internal Medicine, 1975, 135, 1155

12 Berger, M P, and Paz, J, fournal of the American Medical Association, $1976,236,2517$.

13 Schlossberg, D, Brooks, J B, and Shulman, J A, fournal of Clinical Microbiology, 1976, 3, 239.

14 Baker, R D, American fournal of Clinical Pathology, 1976, 65, 83.
15 Menon, A, and Rajamani, R, British Fournal of Diseases of the Chest, 1976, 70, 269.

${ }^{16}$ Fisher, B D, and Armstrong, D, New England fournal of Medicine, 1977, 297, 1440.

${ }^{17}$ Reese, M C, and Colclasure, J B, Archives of Otolaryngology (Chicago), 1975, 101, 698.

${ }^{8}$ Staib, F, et al, Zentralblatt für Bakteriologie, Parasitenkunde, Infektionskrankheiten und Hygiene, I Abt, Originale, 1977, 237, 378.

19 Tolentino, P, and Borrone, C, Scandinavian fournal of Infectious Disease (Stockholm), 1976, 8, 61 .

${ }^{20}$ Robert, F, Durant, J R, and Gams, R A, Archives of Internal Medicine, 1977, 137, 688.

${ }^{21}$ Schupbach, C W, et al, Archives of Dermatology, 1976, 112, 1734.

${ }^{22}$ Appelbaum, E, and Shtokalko, S, Annals of Internal Medicine, 1957, 47, 346.

${ }^{23}$ McGill, P E, et al, East African Medical fournal, 1969, 46, 663.

${ }^{24}$ Utz, J P, et al, Antimicrobial Agents and Chemotherapy, 1969, 8th conf, p 344.

${ }^{25}$ Utz, J P, et al, Journal of Infectious Diseases, 1975, 132, 368.

${ }^{26}$ Tobias, J S, Wrigley, P F, and Shaw, E, Postgraduate Medical fournal, 1976, 52, 305.

\section{Cardiac sarcoidosis}

The commoner clinical presentations of sarcoidosis are easily recognisable and have a good prognosis, but there are important exceptions. Diagnostically, an uncommon site, such as the central nervous system, ${ }^{1}$ the liver, ${ }^{2}$ and the heart, ${ }^{3}$ may cause difficulty. Prognostically, there are two groups of patients whose lives are likely to be shortened. One, fortunately small, consists of those with the special problem of hypercalcaemia, which, unless controlled, causes non-granulomatous nephropathy; the other, larger but still a minority of all cases, includes those with persistent granulomatosis of a vital organ. In these circumstances function may be seriously disturbed either by widespread progressive changes or by the effects of circumscribed granulomatous deposits.

These points have recently been substantiated for cardiac sarcoidosis in a study by Roberts $e t \mathrm{al}^{4}$ based principally on 35 necropsies at the United States National Institutes of Health and Armed Forces Institute of Pathology. Their account provides the fullest description yet available of the varied pathological features of sarcoidosis affecting the heart and its correlation with the similarly varied clinical presentation. The criteria adopted for diagnosing cardiac sarcoidosis were sarcoid-type granulomas in lymph nodes and either similar granulomas in the heart or transmural myocardial scarring without narrowing of coronary arteries. These conform to the definition of sarcoidosis suggested by Scadding ${ }^{5}$ and recently restated by Mitchell et al. ${ }^{6}$

Of the 35 cases, 26 had shown clinically evident effects of cardiac sarcoidosis and nine had shown no such evidence. This difference of clinical presentation corresponded with a striking difference in the extent of morbid changes. In all 26 with cardiac malfunction attributable to sarcoidosis the heart was massively affected. The left ventricular wall was damaged in all cases, and the interventricular septum in 19 and papillary muscles in 17; a left ventricular aneurysm was present in two cases. The right ventricular wall was affected in 12 , and the right and left atria in three and two cases respectively. In all but one of these 26 cases cardiac sarcoidosis was the immediate cause of death. In six, sudden death had been the first evidence of disease, and in 11 more death was sudden after illnesses mostly characterised by ventricular dysrhythmias or heart block. Such abnormalities were observed in 16 of 20 patients in whom electrocardiograms had been recorded; in most of these the changes were gross, with episodes of ventricular tachycardia in five cases and complete heart block in 
six. Eight patients had had congestive heart failure. Two of these had mitral incompetence, and at necropsy were found to have extensive lesions in the papillary muscles. Recurrent pericardial effusions had preceded death in two cases: in both there were granulomas in the left ventricular myocardium as well as the pericardium.

By contrast, in the nine cases in which there had been no symptoms attributable to cardiac sarcoidosis, the heart lesions had consisted of small granulomas, visible only microscopically in eight. Seven had shown evidence of cardiac dysfunction from other causes: cor pulmonale due to extensive pulmonary sarcoidosis in four, valvular disease (presumably rheumatic) in two, and myocardial infarction in one.

The frequency of myocardial sarcoidosis is difficult to estimate. Some reports have stated that a high proportion of patients with sarcoidosis show electrocardiographic changes suggestive of myocardial lesions, ${ }^{78}$ and that these changes may return to normal with regression of the sarcoidosis. ${ }^{8}$ This suggests that in the active phase scattered granulomas may be present in the myocardium in some cases but resolve-as they do in other organs-with the spontaneous regression of the disease that occurs in most cases. Whether or not that is so, few patients with sarcoidosis develop symptomatic cardiac disease. ${ }^{5}$ The difficulty of diagnosis varies greatly with the mode of presentation. Sudden death, presumably from ventricular fibrillation or heart block, in individuals who have had no symptoms leading them to seek medical advice is a problem for the pathologist rather than the clinician. Cases of this sort may be expected to be over-represented in studies based on necropsy reports, but they are frequent in all reported series: there were 12 in the 50 cases of cardiac sarcoidosis collected by Fleming ${ }^{3}$ from clinical records in Britain. In a patient known to have sarcoidosis, the appearance of a ventricular dysrhythmia or heart block of any degree must be strong presumptive evidence that the heart is affected, particularly if there is no reason to suspect any of the commoner cardiac diseases; and in the exceedingly rare event of the sudden development of mitral incompetence sarcoidosis of papillary muscles is a leading possibility. At least one case of this sort has been treated successfully by replacement of the mitral valve. ${ }^{9}$

Diagnosis is much more difficult when the problem is the coexistence of myocardial dysfunction and sarcoidosis. Coronary angiography may help but may be inconclusive. Direct evidence of coronary artery disease does not exclude the possibility of myocardial sarcoidosis; and, while finding normal vessels supports a diagnosis of cardiac sarcoidosis, it cannot exclude other cardiomyopathies. Extensive lung lesions may complicate the diagnostic problem; but Roberts et $a l^{4}$ found that, both in their 26 cases of cardiac sarcoidosis causing cardiac dysfunction and in 63 similar cases reported by others, the initial symptom was most often related to the heart, and symptoms related to other organs were infrequent.

Cardiac sarcoidosis extensive enough to cause symptoms has a poor prognosis. Sudden death, though fortunately it is rare, is frequent, either as the first and only manifestation or after an illness which is generally not prolonged. Of the 26 patients described by Roberts et al, ${ }^{4} 19$ died within 12 months of the first cardiac symptom. Moreover, as with all the persistent chronic forms of sarcoidosis, the results of treatment are unsatisfactory. The suppressive effect of corticosteroids on the granuloma may help in controlling some of the features of the active phase of the disease: arrhythmias, conduction defects, and electrocardiographic evidence of myocardial dysfunction may be favourably affected. But treatment is in no sense curative. Granulomas tend to recur when corticosteroids are withdrawn, and there is no reason to suppose that the proportion of cases in which such recurrence does not occur is higher than might be expected from the lapse of time in the natural course of the disease. Furthermore, corticosteroids are unlikely to have any effect on the fibrotic scarring which is the end point of persistent granulomatosis.

Effective treatment of sarcoidosis in general must continue to await the elucidation of its aetiology. Even when such treat- $\vec{F}$ ment becomes available, cardiac sarcoidosis will present special $\stackrel{?}{\rightarrow}$ problems, both from diagnostic difficulties and from the occurrence of cases in which the first symptom appears only $\frac{\bar{D}}{\omega}$ when the changes are extensive and irreversible, and may be $\bar{\nabla}$ sudden death.

${ }_{1}^{1}$ Delaney, P, Annals of Internal Medicine, 1977, 87, 336. 2 Israel, H L, and Goldstein, R A, Annals of Internal Medicine, 1973, 79,

${ }^{3}$ Fleming, H A, British Heart fournal, 1974, 36, 54.

4 Roberts, W C, McAllister, H A, and Ferrans, V J, American fournal of Medicine, 1977, 63, 86.

5 Scadding, J G, Sarcoidosis. London, Eyre and Spottiswoode, 1967.

${ }^{6}$ Mitchell, D N, et al, fournal of Clinical Pathology, 1977, 30, 395.

Stein, E, et al, Proceedings of VI International Conference on Sarcoidosis, $\overrightarrow{\overrightarrow{0}}$ p 360. Tokyo, University of Tokyo Press, 1974.

${ }^{8}$ Mikhail, J R, Mitchell, D N, and Ball, K P, Proceedings of VI International $\vec{\circ}$ Conference on Sarcoidosis, p 365. Tokyo, University of Tokyo Press, 1974.

${ }^{9}$ Raftery, E B, Oakley, C M, and Goodwin, J F, Lancet, 1966, 2, 360.

\section{Self-poisoning with beta-blockers}

Self-poisoning with beta-blocking drugs is uncommon. The main clinical features include bradycardia, hypotension, lowoutput cardiac failure, and cardiogenic shock. Airways obstruction may also occur and respiratory depression develop as a result of severe circulatory impairment or of a central drug effect. ${ }^{1}$ In severe overdosage the myocardium may become relatively refractory to pharmacological and electrical stimulation and death occurs in asystole.

Variations in the pharmacological properties of the different beta-blockers affect their therapeutic action and predictable adverse effects, ${ }^{2}$ but we do not know how important these individual features are in serious overdosage. ${ }^{1}$ Patients have tolerated therapeutic doses of up to $4 \mathrm{~g}$ propranolol daily ${ }^{3}$ and deliberate overdosage of both practolol ${ }^{4}$ and propranolol ${ }^{5}$ without serious adverse effects. Conversely, circulatory collapse may occur in patients with pre-existing cardiac failure when sympathetic drive is inhibited by even a small dose of a beta-adrenoceptor antagonist. This observation was originally made in a patient given propranolol intravenously, ${ }^{6}$ but it has also been reported after a single $20-\mathrm{mg}$ dose of oxprenolol by mouth. ${ }^{7}$ Apart from the problems posed by the individual drug, other factors that may complicate management include age, obstructive airways disease, diabetes mellitus, and renal or hepatic insufficiency.

Gastric lavage may allow the tablets taken to be identified but is unlikely to be sufficient to prevent serious poisoning unless performed early, because all beta-blocking drugs are absorbed rapidly. Estimating the blood concentrations of beta-blocking drugs may confirm the self-poisoning, but this is of limited value in immediate management. Haemodialysis is unlikely to rid the body of propranolol, ${ }^{8}$ although it has not been tried in gross overdosage. It may be possible to dialyse beta-blocking drugs which are more water soluble and less 